\title{
Physico-Chemical Characters of Pumpkin (Cucurbita moschata Duch.) Ex Poir Genotype against the Melon Fly (Bactrocera cucurbitae) Reveals Resistance Traits in the Terai Region of West Bengal, India
}

\author{
Roshna Gazmer ${ }^{1 *}$, Nripendra Laskar ${ }^{1}$ and Somnath Mandal ${ }^{2}$ \\ ${ }^{1}$ Department of Agricultural Entomology, ${ }^{2}$ Department of Biochemistry, Uttar Banga Krishi \\ Viswavidyalaya, Pundibari, Cooch Behar, West Bengal- 736165, India \\ *Corresponding author
}

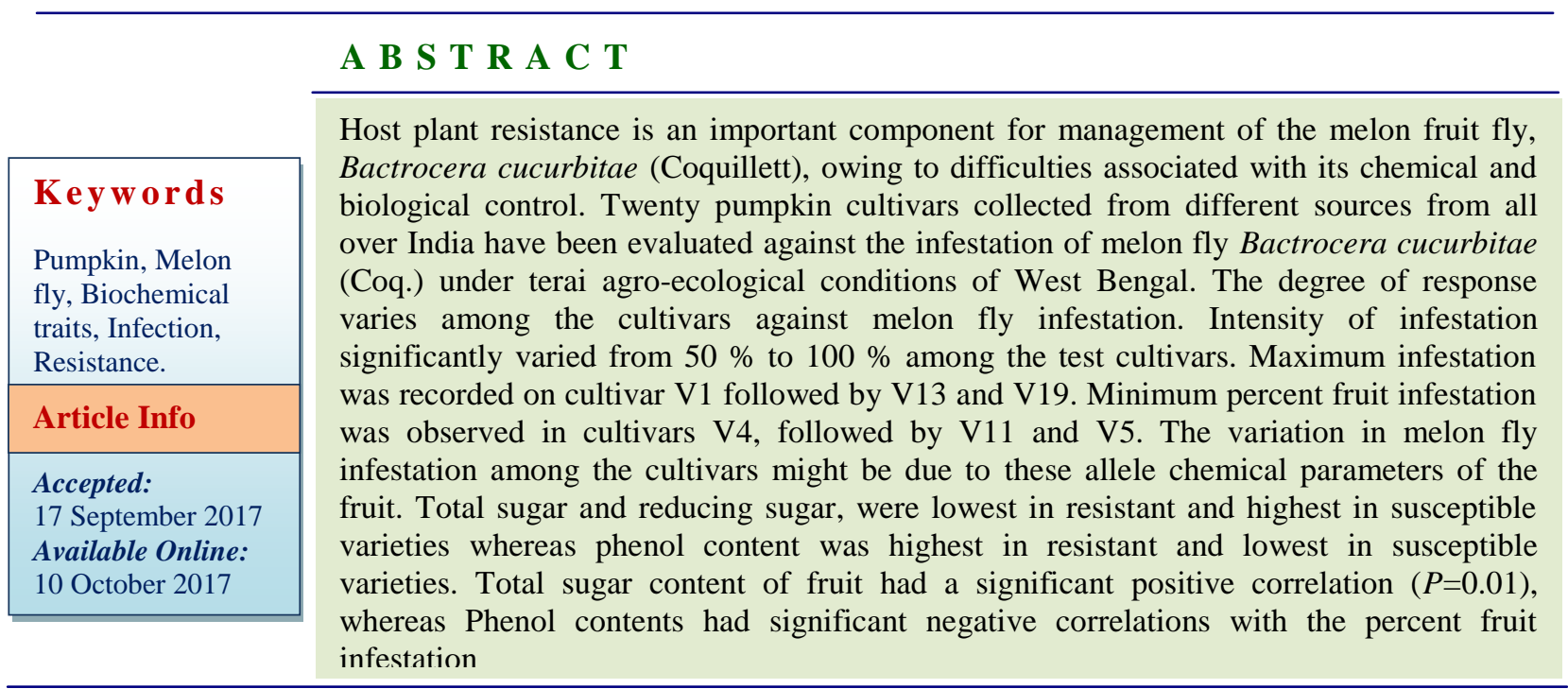

\section{Introduction}

Pumpkin (Cucurbita moschata var Duch. Ex Poir.) is one of the most important cucurbitaceous vegetable crops grown worldwide. Young leaves, flowers, immature and mature fruits of pumpkin are used as vegetable. This cucurbitaceous vegetable suffers severe insect-pest attack due to favourable conditions available for their multiplication and development. The fruit fly, Bactrocera cucurbitae (Coq.) (Diptera: Tephritidae) attacks fruits of the crop and is one of the most important biotic limiting factors. The extent of losses caused by the pest varies from 30-100\% depending on cucurbit species and season (Waterhouse, 1993; Dhillon et al., 2005a). Effective management of this dreaded pest is difficult due to its concealed feeding habit and typical life history. Efforts in fruit fly management were focused mainly on mature adult including bagging of fruits, field sanitation, bait traps, cuelure and sterile insect technique (SIT) (Hendrichs et al., 2002), spray of protein baits with toxicants, growing fruit flyresistant genotypes, augmentative releases of biological control agents, and soft 
insecticides. Conventional insecticides do not work well against the pest, rather, excessive use of pesticides cause development of resistance, resurgence, hazards to non-target organism and environmental pollution. In this perspective, host plant resistance (HPR) is one of the most important and promising aspects of pest management. Plants are generally exposed to a variety of biotic and abiotic factors that may alter their genotypic or phenotypic properties resulting in different mechanisms of resistance which enable plants to avoid, tolerate or recover from the effects of pest attacks (Gogi et al., 2010b; Pedigo 1996; Sarfraz et al., 2006). Such mechanisms of plant resistance have been effectively used against insect pests in many field and horticultural crops (Dhillon et al., 2005b; Gogi et al., 2010a; Kogan 1982; Sarfraz et al., 2007). Mechanisms of resistance in plants are either constitutive or induced (Karban and Agrawal 2002; Painter 1951; Traw and Dawson 2002) and are grouped into three main categories: antixenosis, antibiosis and tolerance (Painter 1951).

Plants responsible for antibiosis resistance may cause reduced insect survival, prolonged developmental time, decreased size and reduced fitness of new generation adults (Gogi et al., 2010b; Painter 1951; Sarfraz et al., 2006, 2007). However, the indigenous cultivars have their innate heritable chemicophysical features to resist the infestation caused by the melon fruit fly. Unfortunately, information regarding the morphological factors such as skin toughness of fruit, skin thickness of fruit (Dhillon et al., 2005b) and chemical factors such as in moisture level; ascorbic acid, reducing sugar, non-reducing sugar and total sugars, nitrogen, protein, phosphorus and potassium contents (Tewatia et al., 1998) etc. responsible for this variation in different levels of infestations is vital for initiating crop improvement program to develop resistant lines. In the present study an initiative was undertaken to find out the impact of different physical and morphological traits of pumpkin fruits on the extent of infestation and field evaluation of pumpkin cultivars against the infestation of melon fruitfly.

\section{Materials and Methods}

\section{Plant materials}

The study was conducted at the Instructional Farm, Uttar Banga Krishi Viswavidyalaya, Pundibari, Cooch Behar, West Bengal, India (26 19'N latitude and 89 23'E longitude and an altitude of $43 \mathrm{MSL})$. Twenty varieties of Pumpkin including local accessions, open pollinated, hybrids were taken for study. The varieties taken for the study were as follows:

V1: Pumpkin collection, Eluru, Andhra Pradesh

V2: Baidyabati Kumra, KrishiMangal Vegetable Seeds, Kolkata

V3: Pumpkin collection, Pantnagar-2, Uttarakhand

V4: Baidyabati, Debgiri seed, Kolkata

V5: Pumpkin collection, Pasighat-1, Arunachal Pradesh

V6: Pumpkin collection, Pundibari Local-1, West Bengal

V7: Pumpkin collection, Pantnagar-1, Uttarakhand

V8: Pumpkin collection, Pundibari Long Variety, West Bengal

V9: Arka Suryamukhi, IIHR, Bangalore

V10: Pumpkin collection, Phek, Nagaland 
V11: Pumpkin collection, Gagan Sardar Para2, Tripura

V12: Pumpkin collection, Trivendrum, Kerala

V13: Pumpkin collection, Gagan Sardar Para1, Tripura

V14: Pumpkin collection, Pasighat-2, Arunachal Pradesh

V15: Pumpkin collection, Komatapalli, Andhra Pradesh

V16: Pumpkin collection, Pundibari Local-2, West Bengal

V17: Pumpkin collection, Ambalavayal, Kerala

V18: Pumpkin collection, Pundibari Local-3, West Bengal

\section{V19: Barahmasi, Varanasi, Uttar Pradesh}

V20: Pumpkin collection, Pundibari Local-4, West Bengal

The test materials were planted on raised beds of $6 \mathrm{~m} \times 3 \mathrm{~m}$ with a plant-to-plant spacing of $3 \mathrm{~m}$ and row to row distance at $3 \mathrm{~m}$ in December (winter season) of 2014 and February (summer season) of 2015. The experiment was replicated thrice arranged by following randomized block design (RBD). In each bed five plants were tagged randomly for recording observation. The winter season crop fruited in April-May and the summer season crop during June-July.

Recommended agronomic practices were adopted to raise the crop except chemical control of insect pests. Marketable sized fruits were picked at three days interval and brought to the laboratory for recording observation regarding physical characteristics and percent fruit infestation. The infested fruits were counted and the percent fruit infestation was calculated both in number and weight basis \% fruit infestation was determined as follows:

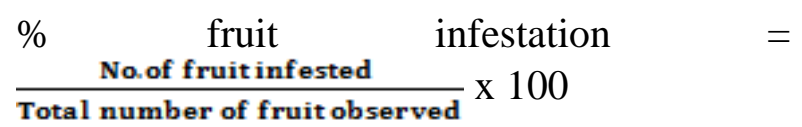

The genotypes were grouped by following the rating system, given by Nath (1966) for the fruit damage as immune (no damage), highly resistant (1-10\%), resistant (11-20\%), moderately resistant (21-50\%), susceptible (51-75\%) and highly susceptible (76-100\%). The infested fruits were cut open to count the number of maggots of melon fly fruit.

\section{Biochemical fruit traits}

Biochemical fruit traits of different pumpkin varieties/genotypes were studied. Three fresh fruits of each genotype were picked from the field randomly at three different stages i.e Early stage (0-10 DAS), Pre-maturity stage (20-25 DAS), Maturity stage (35-40 DAS) and brought to the Biochemistry Laboratory of UBKV, Pundibari, Cooch Behar. The fresh fruits were cut into small pieces. The biochemical contents in fresh fruits were determined following protocols of for Total sugar and Malik and Singh (1980) for Total phenols content.

\section{Statistical analysis}

The data on percentage fruit infestation and biochemical fruit traits were analyzed through one-way ANOVA using SPSS 16 software (O'Connor 2000). The means of significant parameters, among tested varieties/ genotypes, were compared using Tukey's honestly significant difference (HSD) test for paired comparisons at the 5\% probability level. Correlations between biochemical fruit traits and fruit fly parameters (percent fruit infestation) were determined using correlation analysis and backward stepwise multiple regression analysis at the $95 \%$ significance level (Table 3). 


\section{Results and Discussion}

\section{Percent fruit infestation}

Significant differences in percent fruit infestation were observed among the test cultivars in both the seasons (2014-15 and 2015-16) of study with three different stages i.e. Early, Pre-maturity and Maturity stages (Table 1 and Fig. 1).

\section{Early stage of fruit infestation}

Percent fruits infestation by the melon fly at Early stage varied significantly from $12.82 \%$ to $58.35 \%$ (Pooled data 2014-15 and 2015-16) being lowest infestation in V4 and highest in V1. Maximum percent fruit infestation was observed in V1, V13 and V19. Minimum percent fruit infestation was observed in cultivars V4, V11 and V5. Other varieties showed moderate percent fruit infestation.

\section{Pre-maturity stage of fruit infestation}

Percent fruits infested by the melon fly at Prematurity stage varied significantly from $15.97 \%$ to $59.31 \%$ (Pooled data 2014-15 and 2015-16) being lowest infestation in V4 and highest in V1. Maximum percent fruit infestation was observed in V1, V13 and V19. Minimum percent fruit infestation was observed in V4, V11 and V5. Other varieties showed moderate percent fruit infestation.

\section{Maturity stage of fruit infestation}

Percent fruits infested by the melon fly at maturity stage varied significantly from $20 \%$ to $61 \%$ (Pooled data 2014-15 and 2015-16) being lowest infestation in V4 and highest in V1. Maximum percent fruit infestation was observed in cultivars V1, V13 and V19. Minimum percent fruit infestation was observed in V4, V11 and V5. Other varieties showed moderate percent fruit infestation.

\section{Biochemical fruit traits}

Biochemical fruit traits of the pumpkin varieties/ genotypes was evaluated at three different stages of fruit i.e., Early stage (0-10 DAS), Pre- maturity stage (20-25 DAS) and Maturity stage (35-40 DAS) (Table 2).

\section{Total sugar}

Total sugar content in Early stage varied significantly from 183.90 to $316.11 \mathrm{mg} / \mathrm{g}$ (Pooled data 2014-15 and 2015-16) with Maximum Total sugar content during Early stage of fruit observed in cultivars V1 $\left(316.1^{\mathrm{a}}\right), \mathrm{V} 13\left(314.56^{\mathrm{b}}\right)$ and V19 $\left(314.47^{\mathrm{b}}\right)$ and Minimum Total sugar content during Early stage of fruit were observed in V4 $\left(183.90^{\mathrm{q}}\right), \mathrm{V} 11\left(207.16^{\mathrm{p}}\right)$ and V5 $\left(223.57^{\mathrm{o}}\right)$.

Pre-maturity stage varied significantly from 322.02 to $427.84 \mathrm{mg} / \mathrm{g}$ (Pooled data 2014-15 and 2015-16) with Maximum Total sugar content during Early stage of fruit observed in cultivars V1 $\left(427.84^{\mathrm{a}}\right), \mathrm{V} 13\left(324.61^{\mathrm{b}}\right)$ and V19 $\left(272.15^{\mathrm{k}}\right)$ and Minimum Total sugar content during Early stage of fruit observed in V4 $\left(322.02^{\mathrm{c}}\right), \mathrm{V} 11\left(319.83^{\mathrm{d}}\right)$ and $\mathrm{V} 5\left(301.33^{\mathrm{e}}\right)$. Maturity stage varied significantly from 380.48 to $842.97 \mathrm{mg} / \mathrm{g}$ (Pooled data 2014-15 and 2015-16 with Maximum Total sugar content observed in V1 $\left(842.97^{\mathrm{a}}\right), \quad \mathrm{V} 13$ $\left(826.01^{\mathrm{b}}\right)$ and V19 $\left(818.19^{\mathrm{c}}\right)$ and Minimum Total sugar content observed in V4 $\left(380.48^{\mathrm{s}}\right)$, V11 $\left(392.89^{\mathrm{r}}\right)$ and V5 $\left(407.58^{\mathrm{q}}\right)$.

\section{Total phenol}

Total Phenol content in Early stage varied significantly from 401.38 to $1373.32 \mathrm{mg} / \mathrm{g}$ (Pooled data 2014-15 and 2015-16) with Minimum Total Phenol content observed in cultivars V1 $\left(401.38^{\mathrm{k}}\right), \quad \mathrm{V} 13\left(422.97^{\mathrm{kj}}\right)$ and V19(512.39 $9^{\mathrm{ij}}$ ) and Maximum Total phenol content observed in V4(1373.32 $)$, V11 $\left(1290.33^{\mathrm{a}}\right)$ and V5 $\left(1187.03^{\mathrm{b}}\right)$. 
Table.1 Fruit infestation $\%$

\begin{tabular}{|c|c|c|c|c|c|c|c|c|c|c|c|c|}
\hline \multirow[t]{2}{*}{ Varieties } & \multicolumn{4}{|c|}{ Early } & \multicolumn{4}{|c|}{ Pre-maturity } & \multicolumn{4}{|c|}{ Maturity } \\
\hline & 2014 & 2015 & Pooled & $\begin{array}{l}\text { Resistance } \\
\text { category }\end{array}$ & 2014 & 2015 & Pooled & $\begin{array}{l}\text { Resistance } \\
\text { category }\end{array}$ & 2014 & 2015 & Pooled & $\begin{array}{l}\text { Resistance } \\
\text { category }\end{array}$ \\
\hline V1 & $58.29(49.59)^{k}$ & $58.39(49.83)^{\circ}$ & $58.35(49.80)^{b}$ & $\mathrm{~S}$ & $59.30(50.34)^{\mathrm{a}}$ & $59.31(50.35)^{\mathrm{a}}$ & $59.31(50.36)^{\mathrm{a}}$ & $\mathrm{S}$ & $61.08(51.37)^{\mathrm{c}}$ & $60.91(41.29)^{\mathrm{a}}$ & $61.00(51.35)^{\mathrm{a}}$ & $\mathrm{S}$ \\
\hline V2 & $55.00(48.02)^{\circ}$ & $55.00(47.85)^{\mathrm{pq}}$ & $55.00(47.87)^{d}$ & $\mathrm{~S}$ & $56.54(48.71)^{j}$ & $56.41(48.69)^{\mathrm{d}}$ & $56.48(48.72)^{\mathrm{c}}$ & $\mathrm{S}$ & $57.83(49.49)^{f}$ & $57.86(36.99)^{j}$ & $57.85(49.52)^{e}$ & $\mathrm{~S}$ \\
\hline V3 & $45.97(42.62)^{\mathrm{h}}$ & $45.98(42.71)^{\mathrm{i}}$ & $45.98(42.70)^{\mathrm{h}}$ & MR & $41.35(42.58)^{g}$ & $46.27(42.80)^{\mathrm{g}}$ & $43.81(41.66)^{i}$ & MR & $46.12(42.77)^{\mathrm{e}}$ & $46.12(39.12)^{d}$ & $46.12(42.78)^{\mathrm{h}}$ & MR \\
\hline $\mathrm{V} 4$ & $12.85(20.23)^{\mathrm{d}}$ & $12.79(20.94)^{\mathrm{c}}$ & $12.82(20.98)^{\mathrm{p}}$ & $\mathrm{R}$ & $16.01(23.44)^{q}$ & $15.93(23.48)^{9}$ & $15.97(23.56)^{\circ}$ & $\mathrm{R}$ & $20.00(26.53)^{j}$ & $19.97(30.67)^{\mathrm{s}}$ & $20.00(26.57)^{9}$ & $\mathrm{R}$ \\
\hline V5 & $21.28(27.33)^{\text {ef }}$ & $21.13(27.35)^{e}$ & $21.21(27.42)^{\mathrm{n}}$ & MR & $23.18(28.66)^{\mathrm{n}}$ & $21.46(27.46)^{\circ}$ & $23.11(28.73)^{\mathrm{m}}$ & MR & $24.17(29.39)^{i}$ & $24.14(31.64)^{r}$ & $24.16(29.44)^{p}$ & MR \\
\hline V6 & $25.30(29.83)^{\mathrm{f}}$ & $25.28(30.20)^{\mathrm{g}}$ & $25.29(30.19)^{1}$ & MR & $22.79(28.15)^{\mathrm{p}}$ & $23.02(28.61)^{\circ}$ & $22.13(28.06)^{\mathrm{m}}$ & MR & $25.82(30.49)^{\operatorname{tg}}$ & $26.29(32.07)^{\mathrm{p}}$ & $26.06(30.70)^{\circ}$ & MR \\
\hline V7 & $48.93(44.16)^{g}$ & $48.99(44.41)^{\mathrm{h}}$ & $48.96(44.41)^{\mathrm{e}}$ & MR & $49.14(44.53)^{b}$ & $49.10(44.48)^{b}$ & $49.12(44.50)^{\mathrm{de}}$ & MR & $49.99(44.87)^{b}$ & $50(40.65)^{\mathrm{b}}$ & $50.00(45.00)^{f}$ & MR \\
\hline V8 & $47.29(43.35)^{i}$ & $47.04(43.27)^{j}$ & $47.17(43.38)^{g}$ & MR & $47.88(43.75)^{\mathrm{e}}$ & $47.86(43.79)^{f}$ & $47.87(43.78)^{\mathrm{ef}}$ & MR & $48.43(44.12)^{\mathrm{de}}$ & $48.15(38.10)^{f}$ & $48.29(44.02)^{g}$ & MR \\
\hline V9 & $42.38(40.51)^{1}$ & $42.40(40.65)^{\mathrm{n}}$ & $42.39(40.63)^{i}$ & MR & $45.04(42.05)^{1}$ & $45.03(42.13)^{\mathrm{k}}$ & $45.04(42.15)^{\text {hi }}$ & MR & $45.51(42.36)^{\mathrm{m}}$ & $45.54(33.25)^{\circ}$ & $45.53(42.44)^{1}$ & MR \\
\hline V10 & $48.11(43.83)^{j}$ & $48.17(43.95)^{\mathrm{m}}$ & $48.14(43.94)^{f}$ & MR & $49.63(44.83)^{\mathrm{h}}$ & $49.70(44.82)^{i}$ & $49.67(44.81)^{\mathrm{d}}$ & MR & $49.90(44.70)^{f}$ & $49.91(35.67)^{1}$ & $49.91(44.95)^{j}$ & MR \\
\hline V11 & $17.64(24.47)^{\mathrm{a}}$ & $17.66(24.84)^{\mathrm{d}}$ & $17.65(24.84)^{\circ}$ & $\mathrm{R}$ & $19.49(25.53)^{\mathrm{p}}$ & $19.48(26.18)^{\mathrm{p}}$ & $19.49(26.19)^{\mathrm{n}}$ & $\mathrm{R}$ & $20(26.53)^{d}$ & $19.95(30.46)^{t}$ & $19.98(26.55)^{\mathrm{r}}$ & $\mathrm{R}$ \\
\hline V12 & $47.25(43.21)^{\text {gh }}$ & $47.19(43.38)^{i}$ & $47.23(43.41)^{\mathrm{g}}$ & MR & $47.32(43.43)^{d}$ & $47.29(43.44)^{\mathrm{e}}$ & $47.31(43.46)^{\mathrm{fg}}$ & MR & $47.76(43.61)^{\mathrm{C}}$ & $47.76(37.82)^{g}$ & $47.76(43.72)^{i}$ & MR \\
\hline V13 & $58.91(49.17)^{\mathrm{n}}$ & $58.95(50.17)^{\mathrm{p}}$ & $58.94(50.16)^{a}$ & $\mathrm{~S}$ & $58.19(49.60)^{\mathrm{c}}$ & $58.16(49.70)^{b}$ & $58.18(49.71)^{\mathrm{ab}}$ & $\mathrm{S}$ & $60.23(50.91)^{i}$ & $60.25(39.74)^{c}$ & $60.25(50.91)^{b}$ & $\mathrm{~S}$ \\
\hline V14 & $55.19(47.90)^{\mathrm{m}}$ & $55.27(48.04)^{\mathrm{q}}$ & $55.23(48.00)^{d}$ & $\mathrm{~S}$ & $56.50(48.62)^{\mathrm{c}}$ & $56.49(48.73)^{d}$ & $56.50(48.74)^{\mathrm{c}}$ & $\mathrm{S}$ & $57.99(49.65)^{1}$ & $57.99(37.61)^{\mathrm{h}}$ & $58.00(49.60)^{d}$ & $\mathrm{~S}$ \\
\hline V15 & $22.44(28.03)^{\mathrm{e}}$ & $22.44(28.26)^{f}$ & $22.45(28.28)^{\mathrm{m}}$ & MR & $24.64(29.66)^{\circ}$ & $24.58(29.73)^{\mathrm{n}}$ & $24.61(29.74)^{1}$ & MR & $26.02(30.70)^{\mathrm{h}}$ & $26.14(31.95)^{\mathrm{q}}$ & $26.09(30.71)^{\circ}$ & MR \\
\hline V16 & $28.78(32.02)^{b}$ & $28.77(32.43)^{b}$ & $28.78(32.44)^{j}$ & MR & $30.93(33.75)^{k}$ & $30.90(33.76)^{1}$ & $30.92(33.78)^{j}$ & MR & $31.79(34.30)^{\mathrm{a}}$ & $31.84(37.13)^{i}$ & $31.82(34.34)^{\mathrm{m}}$ & MR \\
\hline V17 & $25.97\left(30.40^{\mathrm{lc}}\right.$ & $25.93(30.62)^{\mathrm{a}}$ & $25.95(30.63)^{k}$ & MR & $26.53(30.98)^{\mathrm{m}}$ & $26.46(30.95)^{\mathrm{m}}$ & $26.50(30.98)^{k}$ & MR & $27.26(31.45)^{\mathrm{C}}$ & $27.22(35.29)^{\mathrm{n}}$ & $27.24(31.46)^{\mathrm{n}}$ & MR \\
\hline V18 & $46.18(42.70)^{j}$ & $46.21(42.82)^{k}$ & $46.20(42.82)^{h}$ & MR & $47.75(43.75)^{i}$ & $47.79(43.71)^{\mathrm{h}}$ & $47.78(43.73)^{f}$ & MR & $48.02(43.91)^{f}$ & $48.02(36.83)^{k}$ & $48.02(43.87)^{j}$ & MR \\
\hline V19 & $56.89(48.90)^{\mathrm{n}}$ & $56.99(49.02)^{r}$ & $56.95(48.99)^{c}$ & $\mathrm{~S}$ & $57.73(49.44)^{\mathrm{f}}$ & $57.78(49.47)^{\mathrm{c}}$ & $57.76(49.47)^{\mathrm{bc}}$ & $\mathrm{S}$ & $58.71(50.34)^{k}$ & $58.72(38.85)^{\mathrm{e}}$ & $58.72(50.02)^{c}$ & $\mathrm{~S}$ \\
\hline V20 & $47.29(43.38)^{k}$ & $47.35(43.50)^{1}$ & $47.33(43.47)^{\mathrm{g}}$ & MR & $46.078(42.54)^{1}$ & $46.07(42.75)^{j}$ & $46.08(42.75)^{\text {gh }}$ & MR & $46.02(42.71)^{\text {gh }}$ & $45.99(35.40)^{\mathrm{m}}$ & $46.01(42.71)^{k}$ & MR \\
\hline
\end{tabular}

Values in parenthesis are angular-transformed xR- resistant, MR- moderately resistant, S- susceptible and HS- highly susceptible

Table.2 Biochemical parameters

\begin{tabular}{|c|c|c|c|c|c|c|c|c|c|c|c|c|c|c|c|c|c|c|}
\hline \multirow[t]{2}{*}{ Varieties } & \multicolumn{3}{|c|}{ Total Sugar (mg/g) Early } & \multicolumn{3}{|c|}{ Total Sugar $(\mathrm{mg} / \mathrm{g})$ Pre- maturity } & \multicolumn{3}{|c|}{ Total Sugar ( $\mathrm{mg} / \mathrm{g})$ Maturity } & \multicolumn{3}{|c|}{ Total Phenol (mg/g) Early } & \multicolumn{3}{|c|}{ Total Phenol (mg/g) Pre-maturity } & \multicolumn{3}{|c|}{ Total Phenol (mg/g) Maturity } \\
\hline & 2014 & 2015 & Pooled & 2014 & 2015 & Pooled & 2014 & 2015 & Pooled & 2014 & 2015 & Pooled & 2014 & 2015 & Pooled & 2014 & 2015 & Pooled \\
\hline V1 & $314.17^{\mathrm{a}}$ & $318.18^{\mathrm{a}}$ & $316.1^{\mathrm{a}}$ & $427.18^{\mathrm{a}}$ & $428.50^{\mathrm{a}}$ & $427.84^{\mathrm{a}}$ & $805.08^{\mathrm{a}}$ & $880.85^{\mathrm{a}}$ & $842.97^{\mathrm{a}}$ & $376.88^{g}$ & $425.87^{\mathrm{s}}$ & $401.38^{\mathrm{k}}$ & $460.11^{\mathrm{c}}$ & $498.75^{\mathrm{t}}$ & $479.4^{\mathrm{e}}$ & $526.25^{\mathrm{t}}$ & $608.755^{t}$ & $567.51^{t}$ \\
\hline $\mathrm{V} 2$ & $310.12^{\mathrm{b}}$ & $304.10^{\mathrm{d}}$ & $307.1183^{\mathrm{c}}$ & $324.75^{\mathrm{ab}}$ & $324.46^{\mathrm{b}}$ & $277.32^{j}$ & $762.21^{\mathrm{c}}$ & $804.58^{\mathrm{d}}$ & $783.40^{\mathrm{e}}$ & $564.74^{\mathrm{efg}}$ & $597.26^{\circ}$ & $581.01^{\text {ih }}$ & $712.77^{\mathrm{b}}$ & $505.65^{\mathrm{s}}$ & $609.2^{\mathrm{de}}$ & $793.14^{\mathrm{p}}$ & $638.626^{\mathrm{P}}$ & $715.88^{\mathrm{p}}$ \\
\hline V3 & $249.4^{j}$ & $273.02^{1}$ & $261.2133^{1}$ & $283.21^{\mathrm{h}}$ & $319.43^{f}$ & $322.42^{\mathrm{c}}$ & $544.36^{\mathrm{g}}$ & $773.71^{f}$ & $659.04^{f}$ & $1020.38^{\text {bcd }}$ & $711.88^{\mathrm{m}}$ & $866.14^{\mathrm{ed}}$ & $822.75^{\mathrm{b}}$ & $947.88^{j}$ & $885.3^{\text {cde }}$ & $885.11^{\circ}$ & $711.735^{\circ}$ & $798.43^{\circ}$ \\
\hline V4 & $157.91^{\mathrm{m}}$ & $209.89^{r}$ & $183.9067^{9}$ & $214.19^{\mathrm{m}}$ & $214.79^{t}$ & $322.02^{\mathrm{c}}$ & $368.87^{\mathrm{P}}$ & $392.08^{\mathrm{s}}$ & $380.48^{\mathrm{s}}$ & $1558.07^{\mathrm{a}}$ & $1188.55^{\mathrm{a}}$ & $1373.32^{\mathrm{a}}$ & $1589.62^{\mathrm{b}}$ & $1370.35^{\mathrm{a}}$ & $1480^{\mathrm{a}}$ & $1899.38^{\mathrm{a}}$ & $1693.10^{\mathrm{a}}$ & $1796.25^{\mathrm{a}}$ \\
\hline V5 & $201.72^{k}$ & $245.42^{\mathrm{p}}$ & $223.575^{\circ}$ & $269.05^{\mathrm{k}}$ & $262.79^{\mathrm{p}}$ & $301.33^{\mathrm{e}}$ & $394.68^{\mathrm{n}}$ & $420.46^{9}$ & $407.58^{\mathrm{q}}$ & $1223.55^{\text {bef }}$ & $1150.50^{\mathrm{c}}$ & $1187.03^{\mathrm{b}}$ & $1451.63^{\mathrm{b}}$ & $1347.11^{\mathrm{c}}$ & $1399.4^{\mathrm{ac}}$ & $1513.15^{\mathrm{c}}$ & $1403.51^{\mathrm{c}}$ & $1458.34^{\mathrm{c}}$ \\
\hline V6 & $257.27^{\mathrm{h}}$ & $254.56^{\mathrm{n}}$ & $255.92^{\mathrm{m}}$ & $277.77^{j}$ & $254.58^{\mathrm{q}}$ & $269.13^{1}$ & $467.09^{\mathrm{n}}$ & $558.89^{\mathrm{m}}$ & $512.99^{\mathrm{n}}$ & $632.53^{\text {bcd }}$ & $1118.83^{\mathrm{d}}$ & $875.69^{\mathrm{ed}}$ & $1278.36^{\mathrm{b}}$ & $1067.55^{g}$ & $1173^{\mathrm{c}}$ & $1314.75^{\mathrm{h}}$ & $1265.94^{\mathrm{h}}$ & $1290.35^{\mathrm{f}}$ \\
\hline V7 & $249.76^{j}$ & $277.96^{j}$ & $263.8633^{k}$ & $270.98^{k}$ & $267.27^{\mathrm{n}}$ & $298.16^{\mathrm{f}}$ & $563.76^{\mathrm{f}}$ & $713.45^{\mathrm{g}}$ & $638.61^{\mathrm{h}}$ & $1084.15^{\mathrm{d}}$ & $841.16^{\mathrm{i}}$ & $962.66^{\mathrm{cd}}$ & $1237.13^{\mathrm{b}}$ & $1265.65^{\mathrm{d}}$ & $1251.4^{\mathrm{c}}$ & $1298.7^{i}$ & $1363.75^{\mathrm{i}}$ & $1331.23^{\mathrm{e}}$ \\
\hline $\mathrm{V} 8$ & $286.87^{\mathrm{e}}$ & $274.66^{\mathrm{k}}$ & $280.7667^{\mathrm{g}}$ & $282.14^{\mathrm{hi}}$ & $284.25^{\mathrm{h}}$ & $293.25^{\mathrm{g}}$ & $484.16^{\mathrm{k}}$ & $563.50^{1}$ & $523.83^{\mathrm{m}}$ & $966.77^{\mathrm{e}}$ & $1101.1^{\mathrm{e}}$ & $1033.94^{\mathrm{c}}$ & $1012.65^{\mathrm{b}}$ & $1014.37^{\mathrm{h}}$ & $1013.5^{\text {cde }}$ & $980.57^{\mathrm{m}}$ & $1003.15^{\mathrm{m}}$ & $991.87^{\mathrm{m}}$ \\
\hline V9 & $262.4^{\mathrm{g}}$ & $268.05^{\mathrm{m}}$ & $265.2567^{j}$ & $308.88^{\mathrm{fg}}$ & $272.16^{\mathrm{m}}$ & $290.52^{\mathrm{h}}$ & $514.26^{\mathrm{i}}$ & $685.32^{\mathrm{i}}$ & $599.80^{j}$ & $673.88^{\mathrm{d}}$ & $806.77^{j}$ & $740.33^{g}$ & $1178.05^{\mathrm{b}}$ & $1250.65^{\mathrm{e}}$ & $1214.4^{\mathrm{c}}$ & $1200.1^{j}$ & $1342.62^{j}$ & $1271.36^{\mathrm{g}}$ \\
\hline V10 & $252.65^{\mathrm{i}}$ & $288.18^{\mathrm{g}}$ & $270.4217^{\mathrm{i}}$ & $280.04^{i j}$ & $264.25^{\circ}$ & $290.52^{\mathrm{h}}$ & $564.26^{\mathrm{f}}$ & $632.46 \mathrm{k}$ & $598.37^{\mathrm{j}}$ & $868.62^{\mathrm{a}}$ & $878.05^{\mathrm{g}}$ & $873.34^{\mathrm{ed}}$ & $1083.15^{\mathrm{b}}$ & $900.15^{1}$ & $991.7^{\text {cde }}$ & $886.65^{\mathrm{n}}$ & $978.755^{\mathrm{n}}$ & $932.70^{\mathrm{n}}$ \\
\hline V11 & $178.90^{1}$ & $235.42^{\mathrm{q}}$ & $207.165^{\mathrm{p}}$ & $224.85^{1}$ & $230.97^{\mathrm{r}}$ & $319.83^{\mathrm{d}}$ & $376.61^{\circ}$ & $409.17^{\mathrm{r}}$ & $392.89^{\mathrm{r}}$ & $1417.77^{\mathrm{d}}$ & $1162.88^{\mathrm{b}}$ & $1290.33^{\mathrm{a}}$ & $1475.05^{\mathrm{b}}$ & $1354.88^{\mathrm{b}}$ & $1415^{\text {ac }}$ & $1805.16^{\mathrm{b}}$ & $1436^{b}$ & $1620.58^{\mathrm{b}}$ \\
\hline $\mathrm{V} 12$ & $287.14^{\mathrm{e}}$ & $287.29^{i}$ & $287.2217^{\mathrm{f}}$ & $310.29^{f}$ & $276.18^{\mathrm{j}}$ & $282.32^{\mathrm{i}}$ & $576.37^{\mathrm{e}}$ & $558.89^{\mathrm{m}}$ & $567.63^{\mathrm{k}}$ & $976.86^{\mathrm{fg}}$ & $754.36^{\mathrm{k}}$ & $865.61^{\mathrm{ed}}$ & $1107.86^{\mathrm{b}}$ & $855.83^{\mathrm{m}}$ & $981.9^{\text {cde }}$ & $1137.23^{1}$ & $927.065^{1}$ & $1032.15^{1}$ \\
\hline V13 & $315.18^{\mathrm{a}}$ & $313.95^{\mathrm{b}}$ & $314.565^{\mathrm{b}}$ & $319.17^{\mathrm{de}}$ & $320.48^{\mathrm{e}}$ & $324.61^{b}$ & $803.29^{\mathrm{a}}$ & $848.72^{b}$ & $826.01^{\mathrm{b}}$ & $414.55^{\text {efg }}$ & $431382^{\mathrm{r}}$ & $422.97^{\mathrm{kj}}$ & $530.385^{\mathrm{b}}$ & $564.26^{\mathrm{P}}$ & $547.3^{\mathrm{de}}$ & $595.11^{\mathrm{s}}$ & $640.387^{\mathrm{s}}$ & $617.75^{\mathrm{s}}$ \\
\hline V14 & $316.22^{\mathrm{a}}$ & $312.60^{b}$ & $314.4183^{b}$ & $323.27^{\mathrm{bc}}$ & $321.56^{\mathrm{d}}$ & $220.68^{\circ}$ & $795.64^{b}$ & $784.42^{\mathrm{e}}$ & $790.04^{\mathrm{d}}$ & $557.27^{\mathrm{bc}}$ & $572.60^{\mathrm{p}}$ & $564.94^{i}$ & $609.75^{\mathrm{b}}$ & $547.78^{\mathrm{q}}$ & $578.8^{\mathrm{de}}$ & $655.61^{\mathrm{q}}$ & $706.392^{\mathrm{q}}$ & $681.01^{9}$ \\
\hline V15 & $250.02^{j}$ & $252.54^{\circ}$ & $251.2833^{\mathrm{n}}$ & $275.88^{\mathrm{m}}$ & $265.47^{\mathrm{s}}$ & $270.67^{i}$ & $474.27^{1}$ & $533.17^{\circ}$ & $503.72^{\mathrm{p}}$ & $1195.52^{\mathrm{efg}}$ & $1150.55^{\mathrm{c}}$ & $1173.04^{\mathrm{b}}$ & $881.55^{\mathrm{b}}$ & $1164.26^{\mathrm{f}}$ & $1022.9^{\text {cde }}$ & $1329.77^{\mathrm{g}}$ & $1363.41^{\mathrm{g}}$ & $1346.60^{\mathrm{d}}$ \\
\hline V16 & $264.12 \mathrm{fg}$ & $298.84^{\mathrm{g}}$ & $281.4833^{\mathrm{g}}$ & $280.48^{\mathrm{hji}}$ & $274.16^{1}$ & $266.18^{\mathrm{m}}$ & $496.87^{j}$ & $554.15^{\mathrm{n}}$ & $525.52^{1}$ & $585.35^{\text {ef }}$ & $921.76^{\mathrm{T}}$ & $753.56^{\mathrm{gt}}$ & $1108.35^{\mathrm{b}}$ & $1003.35^{\mathrm{i}}$ & $1055.9^{\mathrm{cd}}$ & $595.11^{\mathrm{k}}$ & $1234.15^{\mathrm{k}}$ & $1204.27^{\mathrm{h}}$ \\
\hline V17 & $265.15^{\mathrm{f}}$ & $291.41^{j}$ & $278.285^{\mathrm{h}}$ & $278.76^{j}$ & $285.87^{\mathrm{g}}$ & $265.92^{\mathrm{m}}$ & $535.76^{\mathrm{h}}$ & $474.86^{\mathrm{p}}$ & $505.31^{\circ}$ & $625.41^{\mathrm{ef}}$ & $877.31^{\mathrm{h}}$ & $751.36^{\mathrm{gt}}$ & $1082.65^{\mathrm{b}}$ & $936.86^{k}$ & $1009.8^{\text {cde }}$ & $655.61^{\mathrm{f}}$ & $1004.12^{f}$ & $1175.20^{\mathrm{j}}$ \\
\hline V18 & $299.72^{\mathrm{d}}$ & $303.15^{\mathrm{e}}$ & $301.4383^{\mathrm{e}}$ & $317.17^{\mathrm{e}}$ & $279.15^{\mathrm{i}}$ & $227.92^{\mathrm{n}}$ & $565.76^{\mathrm{f}}$ & $646.96^{j}$ & $606.36^{\mathrm{i}}$ & $614.82^{\text {efg }}$ & $713.75^{1}$ & $664.29^{\text {gh }}$ & $957.26^{\mathrm{b}}$ & $852.85^{\mathrm{n}}$ & $905.1^{\text {de }}$ & $1329.77^{\mathrm{d}}$ & $954.72^{\mathrm{d}}$ & $1186.67^{1}$ \\
\hline V19 & $314.19^{\mathrm{a}}$ & $314.76^{b}$ & $314.4767^{b}$ & $321.76^{\mathrm{cd}}$ & $322.27^{\mathrm{C}}$ & $272.15^{k}$ & $805.5^{\mathrm{a}}$ & $830.87^{\mathrm{c}}$ & $818.19^{\mathrm{c}}$ & $474.61^{\mathrm{cd}}$ & $550.16^{\mathrm{q}}$ & $512.39^{i j}$ & $555.55^{\mathrm{a}}$ & $542.87^{\mathrm{r}}$ & $549.21^{\mathrm{a}}$ & $1174.38^{\mathrm{r}}$ & $691.25^{\mathrm{r}}$ & $650.01^{\mathrm{r}}$ \\
\hline $\mathrm{V} 20$ & $305.15^{\mathrm{c}}$ & $301.17^{\mathrm{f}}$ & $303.165^{\mathrm{d}}$ & $306.36^{\mathrm{g}}$ & $274.67^{\mathrm{k}}$ & $214.49^{\mathrm{P}}$ & $604.16^{\mathrm{d}}$ & $698.76^{\mathrm{h}}$ & $651.46^{\mathrm{g}}$ & 1007.16 & $684.95^{\mathrm{n}}$ & $846.06^{\mathrm{ef}}$ & $1006.75^{\mathrm{b}}$ & $742.63^{\circ}$ & $874.7^{\text {cde }}$ & $1346.27^{e}$ & $856.41^{\mathrm{e}}$ & $1116.77^{\mathrm{k}}$ \\
\hline
\end{tabular}

Values in parenthesis are angular-transformed 
Table.3 Correlation coefficient (r) between percent fruit infestation with different biochemical fruit traits of pumpkin varieties/ genotypes

\begin{tabular}{|c|c|c|c|c|c|c|c|c|c|}
\hline & TSE & TSP & TSM & TPE & TPP & TPM & EI & PI & MI \\
\hline EI & $.861 * *$ & $.886^{* *}$ & $.912 * *$ & $-.802 * *$ & $-.525 *$ & $-.881 * *$ & 1 & $.995 * *$ & $.994 * *$ \\
\hline PI & $.853 * *$ & $.877 * *$ & $.908 * *$ & $-.798 * *$ & $-.515 *$ & $-.868 * *$ & $.995 * *$ & 1 & $.998 * *$ \\
\hline MI & $.843 * *$ & $.881 * *$ & $.921 * *$ & $-.803^{* *}$ & $-.522 *$ & $-.876 * *$ & $.994 * *$ & $.998 * *$ & 1 \\
\hline
\end{tabular}

** Significant at $P=0.01$ (two-tailed).

*Significant at $P=0.05$ (two-tailed).

Fig.1 Graph showing percentage of fruit infestation at early, pre-maturity and maturity stage

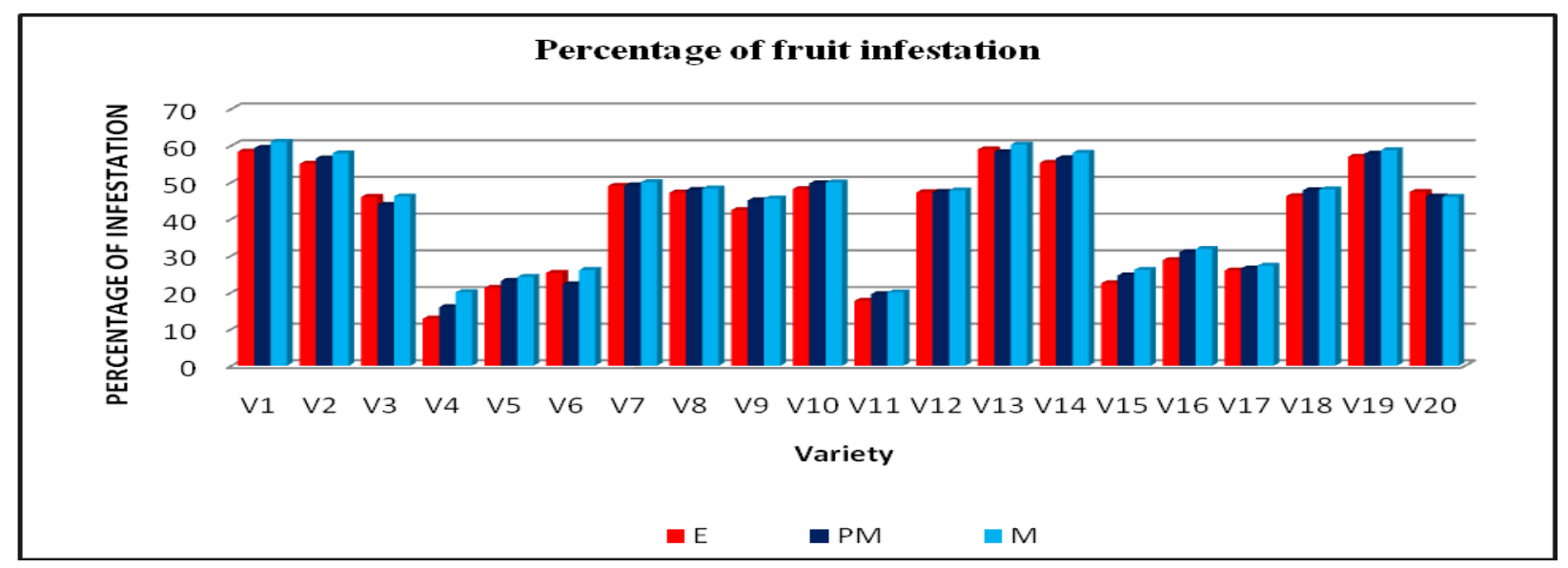

Fig.2 Graph showing

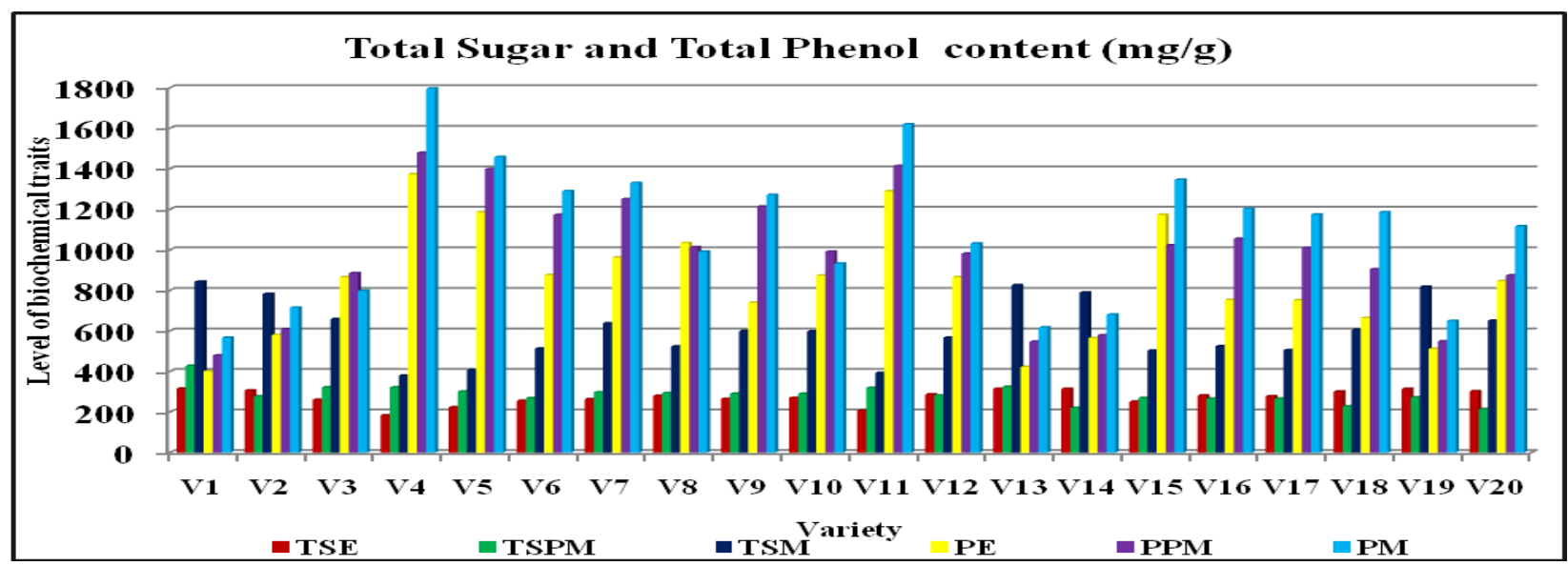

Pre-maturity stage varied significantly from 479.4 to $1480 \mathrm{mg} / \mathrm{g}$ (Pooled data 2014-15 and 2015-16) with Minimum Total Phenol content observed in cultivars V1 $\left(479.4^{\mathrm{e}}\right)$, $\mathrm{V} 13\left(547.3^{\mathrm{de}}\right)$ and V19 $\left(549.21^{\mathrm{a}}\right)$ and Maximum Total phenol content observed in V4 $\left(1480^{\mathrm{a}}\right)$, V11 $\left(1415^{\mathrm{ac}}\right)$ and V5 $\left(1399.4^{\mathrm{ac}}\right)$.
Maturity stage varied significantly from 567.51 to $1796.25 \mathrm{mg} / \mathrm{g}$ (Pooled data 2014-15 and 2015-16) with Minimum Total Phenol content observed in cultivars V1 $\left(567.51^{\mathrm{t}}\right)$, $\mathrm{V} 13\left(617.75^{\mathrm{s}}\right)$ and $\mathrm{V} 19\left(650.01^{\mathrm{r}}\right)$ and Maximum Total phenol content observed in V4 $\left(1796.25^{\mathrm{a}}\right), \quad \mathrm{V} 11\left(1620.58^{\mathrm{b}}\right) \quad$ and 
V5 $\left(1458.34^{\mathrm{c}}\right)$. Other varieties showed moderate content.

EI- Early Infestation \%, PI- Pre-maturity Infestation \%, MI- Maturity Infestation\%, TSE- Total Sugar at Early stage, TSP- Total Sugar at Pre-maturity stage (mg/g), TSMTotal Sugar at Maturity stage $(\mathrm{mg} / \mathrm{g})$, TPETotal Phenol at Early stage $(\mathrm{mg} / \mathrm{g})$, TPPTotal Phenol at Pre-maturity stage $(\mathrm{mg} / \mathrm{g})$, TPM- Total Phenol at Maturity stage $(\mathrm{mg} / \mathrm{g})$.

Total sugar content of fruit at Early stage, Pre-maturity stage and Maturity stage had a highly significant positive correlation $(P=0.01)$ with Early Infestation \%, Prematurity infestation $\%$ and Maturity Infestation \%. Total Phenol content at early stage and Maturity Stage of fruit had a highly significant negative correlation with the Early Infestation \%, Pre- maturity infestation $\%$ and Maturity Infestation \% whereas Total Phenol content at Pre-maturity stage had a significant negative correlation.

Host plant selection by insects is expressed either by the occurrence of a population of insects on the plant in nature or by feeding, oviposition or use of the plant for complete offspring development (Rafiq et al., 2008; Thronsteinson, 1953). Selection is regulated primarily by chemoreception (Gogi et al., 2010b; Jeremy and Szentesi, 2003). Plant varieties/ genotypes possess physiological and biochemical variations due to the environmental stress or genetic makeup, which alter the nutritional values for herbivores (Gogi et al., 2010b; Misirli et al., 2000; Rafiq et al., 2008).

In the present study, Varieties V4 and V11 were the most resistant; and V1, V13, V19, V14 and V2 were susceptible; V3, V5, V6, V7, V8, V9, V12, V15, V16, V17, V18 and V20 were moderately resistant in both seasons. The percent fruit infestation was significantly lower in resistant varieties/ genotypes and higher in susceptible varieties/ genotypes of Pumpkin.

Numerous studies have shown that varieties/ genotypes of the same species could differ significantly in their resistance to insect pests (Dhillon et al., 2005a; Gogi et al., 2009; Sarfraz et al., 2006; Weems and Heppner 2001) and it is caused by biochemical traits of plants.

The allelochemical compounds of fruit were significantly different among the tested pumpkin varieties/ genotypes. Total sugar was lowest in resistant and highest in susceptible varieties/ genotypes, whereas phenol content was highest in resistant and lowest in susceptible varieties/ genotypes. Total sugar, reducing sugar, non-reducing sugar and $\mathrm{pH}$ of fruit had a significant positive correlation, whereas tannins, phenols, alkaloids and flavinoid contents had significant negative correlations with the percent fruit infestation and the larval density per fruit. Sharma and Hall (1971) reported a positive correlation between spotted cucumber beetle (Diabrotica undecimpunctata) feeding and total sugars of various cucurbitaceous crops. In the okra crop, the biochemical characters such as total sugar and crude protein were positively correlated with fruit borer infestation, whereas total phenols were negatively correlated (Ilango and Uthamasamy, 1989; Jat and Pareek, 2003; Sharma and Singh 2010). Similar to our findings, phenols, tannins, and flavonoids enhanced plant defenses against insects (Gogi et al., 2010b; Mila and Scalbert 1994; Ryan and Robards, 1998; TomasBarberan et al., 1988). Reduction of fruit fly infestations on resistant varieties/ genotypes could be due to antibiosis (allelochemicals) and our results suggest that biochemical fruit traits could contribute to these mechanisms of resistance. In summary, certain biochemical 
traits (e.g. total sugar and phenol contents) (Fig. 2) were linked to resistance of Pumpkin against $B$. cucurbitae and therefore can be used as marker traits in plant breeding programs to select resistant varieties/ genotypes.

\section{Acknowledgement}

The authors express their gratitude to Prof. T.K. Hath, Dean Faculty of Agriculture, Uttar Banga Krishi Viswavidyalaya, UBKV, Pundibari, Cooch Behar, West Bengal, India for providing all the facilities. We are also thankful to Department of Biochemistry, UBKV, Pundibari for providing necessary facilities for conducting the experiment.

\section{References}

Dhillon, M.K., Singh R, Naresh JS, Sharma NK 2005b. Influence of physicochemical traits of bitter gourd, Momordica charantia L. on larval density and resistance to melon fly, Bactrocera cucurbitae (Coq.). Journal of Applied Entomology, 129(7): 393399.

Dhillon, M.K., Singh R, Naresh JS, Sharma HC 2005a. The melon fruit fly, Bactrocera cucurbitae: A review of its biology and management. Journal of Insect Science, 5: 40.

Gogi, M.D., Ashfaq M, Arif MJ, Khan MA 2009. Screening of bitter gourd (Momordica charantia) germplasm for sources of resistance against melon fruit fly (Bactrocera cucurbitae) in Pakistan. International Journal of Agricultural Biology, 11: 746-750.

Gogi, M.D., Ashfaq M, Arif MJ, Sarfraz RM, Nawab NN 2010b. Investigating phenotypic structures and allelochemical compounds of the fruits of Momordica charantia L. genotypes as sources of resistance against
Bactrocera cucurbitae (Coquillett) (Diptera: Tephritidae). Crop Protection, 29: 884-890.

Hedge, J.E., Hofreiter BT 1962. In R. L. Whistler and J. N. BeMiller (Eds.), Carbohydrate chemistry 17. New York, NY: Academic Press.

Hendrichs, J., Franz G, Rendon P 1995. Increased effectiveness and applicability of the sterile insect technique through male-only releases for control of Mediterranean fruit flies during fruiting seasons. Journal of Applied Entomology, 119: 371-377.

Ilango, K., Uthamasamy S 1989. Biochemical and physical bases of resistance to bollworms complex in cotton varieties. Madras Agricultural Journal, 76: 73-76.

Jat, K.L., Pareek BL 2003. Biophysical and biochemical factors of resistance in brinjal against Leucinodes orbonalis (Guen). Indian Journal of Entomology, 65: 252-258.

Jeremy, T., Szentesi A 2003. Evolutionary aspects of host plant specialisation - a study on bruchis (Coleoptera: Bruchidae). Oikos, 101: 196-204.

Kogan, M., 1982. Plant resistance in pest management (pp. 93- 134). In R. L. Metcalf and W. H. Luckmann (Eds.), Introduction to insect pest management. New York, NY: John Wiley and Sons.

Mila, I., Scalbert A 1994. Tannin antimicrobial properties through iron deprivation: a new hypothesis. Acta Horticulturae, 381: 749-755.

Misirli, A., Kuden A, Demir G, Gulcan R 2000. Determination of phenolic compounds in some almond hybrids varying in resistance to Pseudomonas amygdala. Report of project TOGTAG1433, pp. 71-86.

Nath, P., 1966. Varietal resistance of gourds to the fruit fly. Indian Journal of Horticulture, 23: 69-78.

O'Connor, B.P., 2000. SPSS and SAS 
programs for determining the number of components using parallel analysis and Velicer's MAP test. Behavior Research Methods, Instruments and Computers, 32: 396-402.

Painter, R.H., 1951. Insect resistance in crop plants. Lawrence, NY, USA: University of Kansas Press.

Pedigo, L.P., 1996. Plant resistance to insects, pp. 413-424. In: Entomology and Pest Management. New Delhi, India: Prentice Hall India Private Ltd.

Rafiq, M., Ghaffar A, Arshad M 2008. Population dynamics of whitefly (Bemisia tabaci) on cultivated crop hosts and their role in regulating its carry-over to cotton. International Journal of Agricultural Biology, 9:6870.

Ryan, D., Robards K 1998. Critical review: phenolic compounds in olives. Analyst, 125: 315-322.

Sarfraz, M., Dosdall LM, Keddie BA 2006. Diamondback moth - host plant interactions: implications for pest management. Crop Protection, 25: 625639.

Sarfraz, M., Dosdall LM, Keddie BA 2007. Resistance of some cultivated Brassicaceae to infestations by Plutella xylostella (Lepidoptera: Plutellidae). Journal of Economic Entomology, 100: 215-224.

Sharma, B.N., Singh S 2010. Biophysical and biochemical factors of resistance in okra against shoot and fruit borer. Indian Journal of Entomology, 72: 212-216.

Tewatia, A.S., Dhankhar BS, Singh R 1998. Evaluation of bitter gourd (Momordica charantia L.) cultivars for resistance to melon fruit fly, Bactrocera cucurbitae (Coquillett). Haryana Journal of Horticultural Science, 27: 266-271.

Thronsteinson, A.J., 1953. The role of host selection in the ecology of phytophagous insects. Canadian Entomology, 85: 276-282.

Tomas-Barberan, F.A., Msonthi JD, Hostettmann K 1988. Antifungal epicuticular methylated flavonoids from Helichrysum nitens. Phytochemistry, 27: 753-755.

Traw, B.M., Dawson T E 2002. Differential induction of trichomes by three herbivores of black mustard. Oecologia, 131: 26-532.

Waterhouse, D.F., 1993. The major arthropod pests and weeds of agriculture in South East Asia. In: ACIAR Monograph 21, pp. 141. Australian Centre for International Agricultural Research, Canberra, Australia.

Weems, H.V., Heppner JB 2001. Melon fly, Bactrocera cucurbitae (Coquillett) (Insecta: Diptera: Tephritidae). Florida Department of Agriculture and Consumer Services, Division of Plant Industry, University of Florid.

\section{How to cite this article:}

Roshna Gazmer, Nripendra Laskar and Somnath Mandal. 2017. Physico-Chemical Characters of Pumpkin (Cucurbita moschata Duch.) Ex Poir Genotype against the Melon Fly (Bactrocera cucurbitae) Reveals Resistance Traits in the Terai Region of West Bengal, India. Int.J.Curr.Microbiol.App.Sci. 6(10): 2023-2031. doi: https://doi.org/10.20546/ijcmas.2017.610.239 